\title{
From divination to madness: features of acute intoxication with Salvia use
}

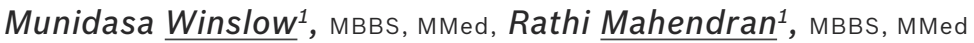

ABSTRACT Salvia divinorum is a psychoactive botanical plant that is increasingly used for the 'legal' highs that it can produce. It is readily available for purchase on the Internet, and most abusers are unaware of the toxicity and abuse potential associated with its use. As the use of novel compounds among abusers is not uncommon, physicians need to increase their awareness and recognition of these new substances. Herein, we report a case of an acute presentation of Salvia intoxication.

Keywords: psychiatric symptoms, Salvia divinorum

\section{INTRODUCTION}

Salvia divinorum, also known as Diviner's Sage or Seer's Sage, is a psychoactive botanical plant used in the religious rituals of some South American countries for its dissociative, hallucinatory, and vision-producing effects. The visions produced have been reported to be mild, short-lasting and mainly religious in nature, in keeping with the situations in which they are predominantly used. $^{(1)}$

Recently, however, substance abusers have been using this plant to achieve 'legal' highs, as it is readily available for purchase on the Internet and is largely unregulated. Abusers generally consider the plant as having low toxicity and a low addictive potential. The plant made its first documented appearance in Singapore in 2003, when some dried plant material analysed in the Narcotics Laboratory at the Centre for Forensic Science, Health Sciences Authority, Singapore, was found to contain the active component salvinorin A. ${ }^{(2)}$ Online searches today reveal many posts about Salvia, including information about its use. Unfortunately, there is scarce information about its psychoactive effects in the medical literature. A PubMed literature search yielded four reports on the psychiatric sequelae of Salvia use, with none of the four reports describing acute phase experiences. We thus report the acute phase experiences of a patient after smoking Salvia. Although the patient's experiences were brief in duration, the experiences were terrifying and unusual compared to those previously reported.

\section{CASE REPORT}

Our patient, a man in his thirties, purchased Salvia through a website on the Internet, where it was sold as 'plant food' and in packets labelled according to four 'strengths' 0 , 5, 10 and 20. He smoked a small amount of the ' $10^{\prime}$ ' strength herb in a joint, and experienced vivid visual and auditory hallucinations that lasted for 12-14 mins. The hallucinations included extracampine hallucinations of a female presence in the room, elementary- and second-person type auditory hallucinations, and pareidolic experiences, with inanimate objects such as furniture coming alive, talking to him and walking about the room. He also had visual hallucinations of ghostly figures talking to him and experienced extreme fear. His consciousness was intact throughout the experience. Despite this experience, the patient decided to try the ' 0 ' strength herb half an hour later, hoping for a 'high' without the vivid hallucinatory effects. However, he re-experienced similar symptoms that were not of lesser intensity or shorter duration. The patient discarded all the packets after both experiences. There were no reported after-effects, and in the longer term, the patient did not experience any perceptual disturbances after his exposure. Although the patient had a history of drug abuse, he had not experienced similar psychopathological symptoms with other drugs.

\section{DISCUSSION}

To the best of our knowledge, the present report is the first report to describe the vivid hallucinatory experiences associated with the use of Salvia. Psychological experiences in acute intoxication with Salvia have not been previously reported, possibly because these experiences are brief. Furthermore, clinicians may be less familiar with the newer products that abusers are purchasing and using, such as this psychoactive plant that has hallucinogenic potential. Patients may not voluntarily mention the use of these substances, or the effects experienced, if not specifically questioned.

One report of Salvia use described the development of acute psychosis and paranoia that necessitated antipsychotic treatment. ${ }^{(3)}$ However, the hallucinatory experiences of the patient in that case were not reported. Furthermore, the subsequent evolution of the patient's illness indicated the patient was already at risk for schizophrenia. ${ }^{(3)}$ Another reported

${ }^{1}$ Department of Psychological Medicine, National University Health System, Singapore

Correspondence: Dr R Mahendran, Senior Consultant Psychiatrist, Department of Psychological Medicine, National University Health System, NUHS Tower Block, Level 9, 1E Kent Ridge Road, Singapore 119228. medrm@nus.edu.sg 
case involved an adolescent who had symptoms of paranoia, déjà vu experiences, and a blunted affect with thought blocking and slow speech that lasted for three days. ${ }^{(4)}$ In another reported case, bizarre feelings of depersonalisation were observed in the patient. ${ }^{(5)}$ Antidepressant effects have also been reported. ${ }^{(6)}$ It appears that the medium-term effects of Salvia use are not associated with hallucinatory experiences, which are more likely a feature in acute intoxication.

The psychoactive component of Salvia is salvinorin A. Although salvinorin $\mathrm{A}$ is a potent and selective kappa-opioid and dopamine (D2) receptor agonist, its psychotomimetic effects are reported to be similar to serotonergic agonists and $\mathrm{N}$-methyl-D-aspartate glutamate receptor antagonists such as mescaline and lysergic acid diethylamide. ${ }^{(7)}$ Behavioural studies involving Salvia users have found that their experiences with Salvia use are similar to those with marijuana use. ${ }^{(8)}$ Laboratory studies with rats have revealed depressive-like effects with salvinorin $\mathrm{A}^{\left({ }^{(9)}\right.}$ and animal studies have shown a rapid onset of action, as well as short distribution and elimination half-lives. ${ }^{(10)}$

In the indigenous use of Salvia, leaf juices are extracted from the fresh leaves of the Salvia plant and mixed with water to create an infusion that is taken orally. The Salvia that was sold as 'plant food' to our patient could have been adulterated or affected by processing, contributing to the psychological effects experienced by the patient. In fact, based on our patient's experiences with the Salvia purchased, the Salvia that had differently labelled strengths could have actually been the same product labelled differently for pricing reasons. It is important to note that the actual substance smoked by the patient was not analysed in the present case. However, the acute onset and ultra-short duration of symptoms is in keeping with the pharmacokinetic profile of salvinorin A. ${ }^{(11)}$ The low costs of using a botanical plant increases the likelihood that the 'plant food' used by our patient did contain Salvia rather than chemically produced compounds.

This report highlights the acute phase symptoms that abusers may experience with seemingly innocuous-sounding substances such as 'plant food'. As an increasing number of abusers are turning to novel compounds to achieve highs, clinicians need to raise their level of awareness of novel drugs and have high levels of suspicion of possible drug abuse, even when blood and urine toxicology tests are negative.

\section{REFERENCES}

1. Valdés LJ 3rd, Díaz JL, Paul AG. Ethnopharmacology of the ska Maria Pastora (Salvia divinorum, Epling and Játiva-M.). J Ethnopharmacol 1983; 7:287-312.

2. Lim M, Tan YY, Lee TK. Unusual Seizures in Singapore. Abstract. Available at: www.hsa.gov.sg. Accessed February 28, 2013.

3. Przekop P, Lee T. Persistent psychosis associated with Salvia divinorum use. Am J Psychiatry 2009; 166:832.

4. Singh S. Adolescent salvia substance abuse. Addiction 2007; 102:823-4.

5. Bucheler R, Glerter $\mathrm{CH}$, Schwoeter P, Gaertner I. Use of non-prohibited hallucinogenic plants: increasing relevance for public health? Phramacopsychiatry 2005; 38:1-5.

6. Hanes KR. Antidepressant effects of the herb Salvia divinorum: a case report. J Clin Psychopharm 2001; 21:634-5.

7. Prisinzano TE. Psychopharmacology of the hallucinogenic sage Salvia. Life Sci 2005; 78:527-31.

8. Albertson DN, Grubbs LE. Subjective effects of Salvia divinorum: LSD- or marijuana-like? J Psychoactive Drugs 2009; 41:213-7.

9. Carlezon WA Jr, Béguin C, DiNieri JA, et al. Depressive-like effects of the kappa-opiod receptor agonist salvinorin $A$ on behavior and neurochemistry in rats. J Pharmacol Exp Ther 2006; 316:440-7.

10. Grundmann O, Phipps SM, Zadezensky I, Butterweck V. Salvia divinorum and salvinorin A: an update on pharmacology and analytical methodology. Planta Med 2007; 73:1033-46.

11. Hooker JM, Xu Y, Schiffer W, et al. Phatmacokinetics of the potent hallucinogen salvinorum $A$ in primates parallels the rapid onset and short duration of effects in humans. Neurolmage 2008; 41:1044-50. 